\title{
A Factorization Approach to Sensitivity Loop Shaping for Disturbance Rejection in Hard Disk Drives
}

\author{
Jinchuan Zheng ${ }^{1}$, Minyue $\mathrm{Fu}^{1}$, Fellow, IEEE, Chunling Du² , Youyi Wang ${ }^{3}$, and Lihua Xie ${ }^{3}$, Fellow, IEEE \\ ${ }^{1}$ School of Electrical Engineering and Computer Science, The University of Newcastle, Callaghan, NSW 2308, Australia \\ ${ }^{2}$ Agency for Science, Technology and Research (A*STAR), Data Storage Institute, Singapore 117608 \\ ${ }^{3}$ School of Electrical and Electronic Engineering, Nanyang Technological University, Singapore 639798
}

\begin{abstract}
This paper investigates a factorization approach to sensitivity loop shaping for disturbance rejection in hard disk drives (HDDs). The advantage of the factorization approach is that the system sensitivity function can be expressed explicitly in terms of a unique design parameter. This greatly simplifies the control design process to make the system sensitivity function match a chosen target sensitivity function with guaranteed stability. By decomposing the controller structure, the design parameter is revealed to behave as a plug-in disturbance filter, the design of which is then presented to suppress the dominant disturbances at some specific frequencies. It is also shown that based on the nominal control system the proposed disturbance filter can reduce the sensitivity gains at specific frequencies without worsening the neighbouring sensitivity gains. Simulation together with implementation results demonstrate that the proposed method can effectively suppress the disturbances around the servo bandwidth and accordingly offers a superior tracking accuracy in comparison with other existing filters.
\end{abstract}

Index Terms-Coprime factorization, disturbance filter, hard disk drive, positioning control.

\section{INTRODUCTION}

$\mathbf{T}$ HE areal density must be increased to meet the ever increasing demands for high-capacity hard disk drives (HDDs) [1]. This requires the head positioning servomechanism of the HDDs to provide positioning accuracy within a few nanometers for the read/write (R/W) heads to follow the very narrow tracks. In HDDs, the track misregistration (TMR) is composed of many factors such as the repeatable runout (RRO) and the nonrepeatable runout (NRRO). The spectral components of RRO appear at the spindle rotation frequency and its harmonics. Major sources of RRO include disk warping, disk slip, thermal expansion of the disks and the written-in TMR while servo writing. The spectral components of NRRO contain broad-band spectra and narrow-band spectra with sharp peaks. Major sources of NRRO include spindle bearing runout, disk flutter, actuator windage due to air turbulence, external shock and vibration, and pivot friction force.

Two main approaches are utilized to minimize the TMR caused by various disturbance sources. One is to increase the servo bandwidth and the other is to suppress the specific disturbances [2]. Ideally, a higher-bandwidth servo can suppress a wider range of disturbances. However, the servo bandwidth is limited by the mechanical resonances of the actuator. Therefore, in recent years there has been intensive research on suppressing the narrow-band disturbances around or above the open-loop 0-dB crossover frequency because such disturbances are becoming another dominant TMR source. For this goal, many specific filters have been reported such as the high-gain filter [3], the phase-stabilized controller [4], the phase-lead peak filter [5]-[7], the resonant filter [8], [9], the time-varying group filter [10], the adaptive peak filter [11], [12], and those developed by using various control theories [13]-[16]. The

Manuscript received April 12, 2009; accepted January 11, 2010. First published March 08, 2010; current version published April 21, 2010. Corresponding author: J. Zheng (e-mail: Jinchuan.Zheng@ @ewcastle.edu.au).

Color versions of one or more of the figures in this paper are available online at http://ieeexplore.iee.org.

Digital Object Identifier 10.1109/TMAG.2010.2040921 design of such disturbance filters is not straightforward because if not properly designed, whether the control system is liable to be unstable or the error sensitivity curve of the feedback loop is severely humped at some unexpected frequencies, which indicates that other disturbances therein would be adversely amplified.

In this paper, a factorization approach is employed for trackfollowing control due to its facility to obtain a desired target sensitivity function in terms of a unique design parameter. By decomposing the controller structure, we further reveal some instructive properties of the control system and address a design method for the disturbance filter to suppress the narrow-band disturbances at some specific frequencies. Compared with our previous filter design [5]-[7], the proposed design method in this paper has two improvements: 1) Multiple disturbances can be suppressed in a unified design process with guaranteed stability. 2) Based on the error sensitivity curve of the nominal feedback loop, the resultant hump due to inserting the disturbance filter is evenly distributed along the high-frequency region only, where the disturbances are normally much less. This improvement is useful because most of the existing filters based on classical loop shaping design usually have to compromise with worsening the sensitivity function's gains at the neighbouring disturbance frequencies, e.g., [3], [5], [8]. In addition, compared with the KYP-based design method [16] for loop shaping, the proposed design process is relatively simple and the derived disturbance filter is more easily implementable.

To verify the efficacy of the proposed method, simulation analysis and experiments on a real HDD are conducted. The results demonstrate that the dominant disturbances around the servo bandwidth can be effectively suppressed, leading to a remarkable reduction of the $3 \sigma$ value of the position error signal (PES). In addition, we compare the performance between the proposed filter and two other existing filters to further justify the advantage of the proposed filter for sensitivity loop shaping.

\section{Plant Model}

Fig. 1 shows the experimental setup of a HDD head positioning system. It consists of a voice coil motor (VCM), a flexure 


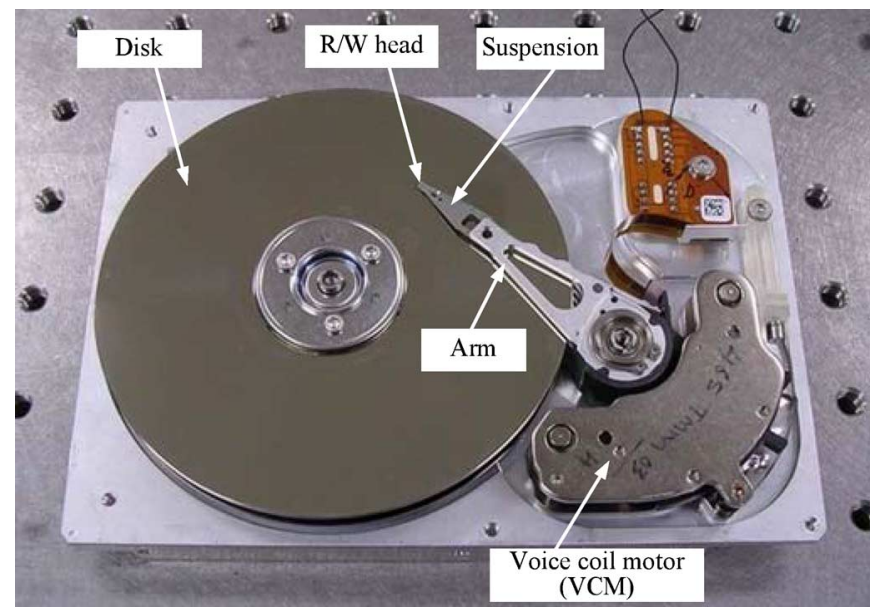

Fig. 1. An experimental HDD head positioning system.

arm and a suspension carrying the $\mathrm{R} / \mathrm{W}$ head. In this setup, the control input is applied to the VCM via a power amplifier. The head position is measured using a laser Doppler vibrometer (LDV). A dynamic signal analyzer (DSA) is used to generate the swept-sinusoidal excitation signals and collect the frequency response data from the head position output to the excitation signals for plant identification purpose. The dashed lines in Fig. 2(a) show the measured frequency responses from the control input to the head position. It is clear that the primary resonance mode that contributes significantly to the head off-track motion is at $4480 \mathrm{~Hz}$, which is caused by the flexibility of the pivot and the VCM-arm assembly. This is the principle mode that limits the servo bandwidth. To identify the VCM plant model, we employ the following transfer function:

$$
G_{V}=K_{V} \sum_{i=1}^{4} \frac{r_{i}}{s^{2}+2 \zeta_{v i} \omega_{v i} s+\omega_{v i}^{2}}
$$

where $K_{V}=2.6 \times 10^{7}$ is the loop gain consisting of an amplifier gain, a torque gain, and an LDV gain, and the other modal parameters are listed in Table I. The solid lines in Fig. 2(a) show that the identified VCM model $G_{V}$ can match the measured model well.

In order to actively damp the resonance modes, we use a resonance compensator of the following form:

$$
F_{V}=\prod_{i=1}^{3} \frac{s^{2}+\zeta_{f 1 i} \omega_{f i} s+\omega_{f i}^{2}}{s^{2}+\zeta_{f 2 i} \omega_{f i} s+\omega_{f i}^{2}}
$$

where $\omega_{f i}$ denotes the resonance frequency, $\zeta_{f 1 i}$ and $\zeta_{f 2 i}$ are the damping ratios chosen to notch the resonance peak. We have implemented the $F_{V}$ to moderately damp the resonance modes at 4.48,6.1, and $8.8 \mathrm{kHz}$ such that the compensated VCM model involves no severe phase lag within $2 \mathrm{kHz}$ while the compensated modes cause insignificant vibrations. Fig. 2(b) shows that the measured frequency responses of the VCM actuator after resonance compensation can actually be approximated as a simple double integrator within $2 \mathrm{kHz}$. Hence, for control design purpose we use the approximated plant model $G$ as follows:

$$
G=G_{V} F_{V} \approx \frac{k}{s^{2}}
$$
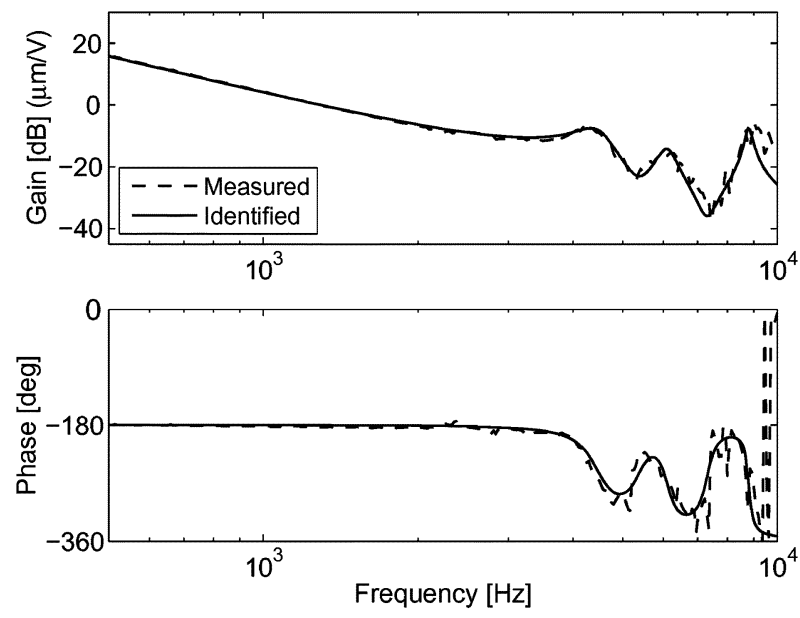

(a)
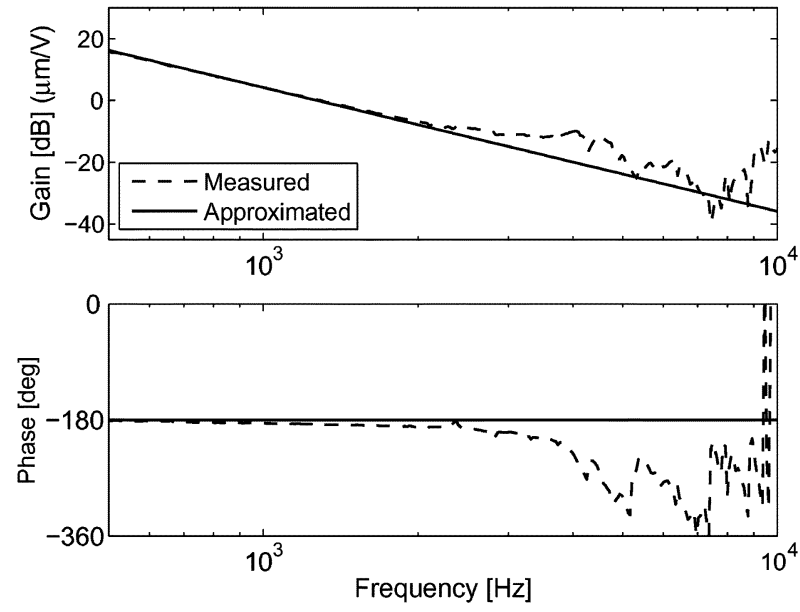

(b)

Fig. 2. Frequency responses of the HDD system. (a) VCM actuator. (b) VCM after resonance compensation.

TABLE I

MODAl PARAMETERS OF $G_{V}(s)$

\begin{tabular}{|c|c|c|}
\hline$r_{1}=2.3$ & $\zeta_{v 1}=0$ & $\omega_{v 1}=0$ \\
\hline$r_{2}=-1.8$ & $\zeta_{v 2}=0.08$ & $\omega_{v 2}=2 \pi 4480$ \\
\hline$r_{3}=-0.8$ & $\zeta_{v 3}=0.04$ & $\omega_{v 3}=2 \pi 6100$ \\
\hline$r_{4}=-1.5$ & $\zeta_{v 4}=0.015$ & $\omega_{v 4}=2 \pi 8800$ \\
\hline
\end{tabular}

where $k=6.4 \times 10^{7}$.

\section{CONTROLler PARAmeterization}

The purpose of this paper is to develop a track-following control system as shown in Fig. 3, where $G$ denotes the VCM model, $K$ the controller to be designed, and $r, u$, pes, and $d$ represent, respectively, the reference signal, the control input, PES, and the disturbances. The reference signal is set as $r=0$ to specify a desired track center. The control objective is to provide accurate head positioning over the track center, whose performance is evaluated by three times the statistical standard deviation of the PES (i.e, PES $3 \sigma$ ). In the sequel, we shall apply a factorization approach to the control design because the resultant sensitivity function can be explicitly expressed in terms of 


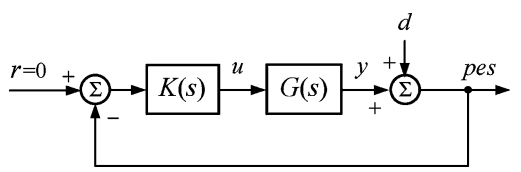

Fig. 3. A HDD track-following control system. $u$ : control input; $d$ : lumped input/output disturbances; $y$ : head position; pes: position error signal; $K(s)$ : controller; $G(s)$ : VCM model.

a unique design parameter and thus facilitating the sensitivity curve shaping for suppressing the specific disturbances around the servo bandwidth with guaranteed stability.

Let $\mathcal{R} \mathcal{H}_{\infty}$ denote the set of all stable, proper, rational transfer function matrices. Let also the right and left coprime factorizations of $G$ be given by

$$
G=N D^{-1}=\tilde{D}^{-1} \tilde{N}
$$

where $N, D, \tilde{N}, \tilde{D} \in \mathcal{R H}_{\infty}$ and satisfy the doubly Bezout identity

$$
\left[\begin{array}{cc}
\tilde{X} & -\tilde{Y} \\
-\tilde{N} & \tilde{D}
\end{array}\right]\left[\begin{array}{ll}
D & Y \\
N & X
\end{array}\right]=I
$$

for some $X, Y, \tilde{X}, \tilde{Y} \in \mathcal{R H}_{\infty}$. Such a factorization can be easily achieved in terms of its state-space realization [17]. To do this, we first represent the plant model $G$ in (3) as a state-space form:

$$
G=C(s I-A)^{-1} B
$$

where

$$
A=\left[\begin{array}{ll}
0 & 1 \\
0 & 0
\end{array}\right], B=\left[\begin{array}{l}
0 \\
k
\end{array}\right], C=\left[\begin{array}{ll}
1 & 0
\end{array}\right]
$$

Since the pairs $(A, B)$ and $(A, C)$ are stabilizable and detectable, respectively, we then select $F$ and $L$ such that $(A-B F)$ and $(A-L C)$ are both Hurwitz. Thus, according to [17], a coprime factorization of $G$ is given by

$$
\left\{\begin{array}{l}
N(s)=C(s I-A+B F)^{-1} B \\
D(s)=I-F(s I-A+B F)^{-1} B \\
\tilde{N}(s)=C(s I-A+L C)^{-1} B \\
\tilde{D}(s)=I-C(s I-A+L C)^{-1} L \\
X(s)=I+C(s I-A+B F)^{-1} L \\
\tilde{X}(s)=I+F(s I-A+L C)^{-1} B \\
Y(s)=-F(s I-A+B F)^{-1} L \\
\tilde{Y}(s)=-F(s I-A+L C)^{-1} L .
\end{array}\right.
$$

According to [18], the class of all linear internally stabilizing controllers $K$ can be parameterized by

$$
K(s)=(D R-Y)(X-N R)^{-1}
$$

where $R \in \mathcal{R H}_{\infty}$ is the free parameter to be designed. By substituting the controller $K$ and the factorized plant model (4) into Fig. 3, we can represent the sensitivity function from $d$ to pes by

$$
\begin{aligned}
S & =(1+G K)^{-1} \\
& =(X-N R) \tilde{D} .
\end{aligned}
$$

We can see that it is advantageous that $S$ is expressed by the unique design parameter $R$ explicitly. This gives a direct relationship between the design parameter and the sensitivity function that reflects the capability of disturbance rejection. Thereby, we can arbitrarily choose a suitable $R \in \mathcal{R} \mathcal{H}_{\infty}$ for desired disturbance rejection with guaranteed system stability.

For easy implementation, the lumped controller (8) is typically decomposed into the controller structure as shown in Fig. 4. The decomposed controller is equivalent to (8) and is separated into two main elements, respectively, referred to as nominal controller and disturbance filter in this paper. Either of the elements is numerically easy to compute and only appears once in the controller. Hence, such a controller structure is preferable in real-time implementation owing to its reduced computation time and improved computation accuracy. Moreover, it offers a straight implication to sensitivity curve shaping for specific disturbances rejection. To see this, we rewrite (9) as

$$
\begin{aligned}
S & =S_{N} S_{R} \\
S_{N} & =X \tilde{D} \\
S_{R} & =1-\frac{N}{X} R
\end{aligned}
$$

where $S_{N}$ represents the sensitivity function of the nominal control system, and $S_{R}$ denotes the sensitivity contribution resulting from $R$. It is shown that $S$ is simply the multiplication of $S_{N}$ and $S_{R}$, and particularly, $S_{N}$ is only determined by $F$ and $L$ instead of involving $R$. Therefore, this property allows a simple two-step design procedure:

S1) Choose vectors $F$ and $L$ such that the nominal controller

$$
K_{N}(s)=\frac{Y}{X}
$$

which is derived by setting $R=0$ in (8), offers basic stability margin and disturbance rejection capability indicated by $S_{N}$.

S2) Design $R$ such that the resultant $S_{R}$ matches a desired sensitivity curve for suppressing the specific disturbances that dominate the tracking inaccuracy of the nominal control system.

Note that in this design strategy, the nominal controller is only capable of rejecting low-frequency disturbances because the servo bandwidth has to be limited to provide sufficient stability margin. Nevertheless, the disturbance filter $R$ is capable of rejecting the disturbances beyond the servo bandwidth. In the following, we shall focus on the design of the disturbance filter 


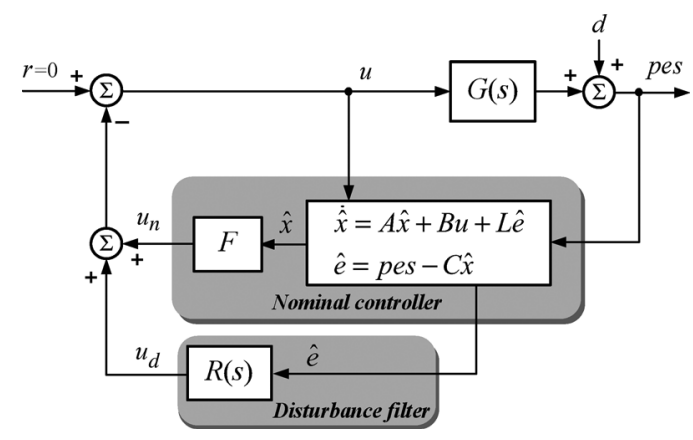

Fig. 4. A decomposed controller structure for easy implementation.

assuming that the nominal controller has been appropriately designed.

\section{Disturbance Filter Design}

In HDDs, the disturbances caused by disk flutter and air turbulence are becoming one of the major TMR sources [19]. Typically, they exhibit several sharp peaks in the power spectra of the PES in the range of $500 \mathrm{~Hz}$ and above. This range is around the servo bandwidth that most of the HDD servo systems now have. Therefore, to suppress these narrow-band disturbances the characteristics of $S_{R}$ should have sufficient low gains at these disturbance frequencies. For instance, a simple target sensitivity function that can express such characteristics is given by

$$
S_{R}^{d}(s)=\prod_{i=1}^{n} \frac{s^{2}+2 \zeta_{1 i} \omega_{i} s+\omega_{i}^{2}}{s^{2}+2 \zeta_{2 i} \omega_{i} s+\omega_{i}^{2}}, \quad \zeta_{1 i}<\zeta_{2 i}
$$

where $n$ is the number of target disturbances for rejection, $\zeta_{1 i}, \zeta_{2 i} \in(0,1)$ are the damping ratios, and $\omega_{i}$ is the disturbance frequency. Apparently, the gains of $S_{R}^{d}$ can be arbitrarily low at the disturbance frequencies by selecting appropriate pair $\left(\zeta_{1 i}, \zeta_{2 i}\right)$.

Substitute (14) into (12), it is straightforward to get an analytic solution of $R=(X / N)\left(1-S_{R}^{d}\right)$, which is stable because $N$ in our case is minimum phase. However, such a $R$ is improper because $X / N$ has a relative degree (excess of poles over zeros) of -2 . In order to make $R$ proper, we can approximate $R$ as

$$
R=\frac{X}{N} Q(s)\left(1-S_{R}^{d}\right)
$$

where $Q(s)$ is a low-pass filter that needs to satisfy two conditions:

1) $\Upsilon(Q) \geq 2$, where $\Upsilon$ denotes the relative degree;

2) $Q(j \omega) \approx 1$ for any $\omega \in\left[0, \omega_{b}\right]$, where $\omega_{b}$ denotes the frequency bandwidth of $Q$.

As such, we can verify $R$ in (15) is both stable and proper. Substitute (15) into (12), we have

$$
S_{R}=1-Q(s)+Q(s) S_{R}^{d}(s) .
$$

Since $Q(j \omega) \approx 1$ in the specified frequency bandwidth, the target sensitivity function can be approached as

$$
S_{R}(j \omega) \approx S_{R}^{d}(j \omega), \quad \forall \omega \in\left[0, \omega_{b}\right] .
$$

By far, we have the following remarks with respect to the design of $R$ :

1) The proposed disturbance filter $R$ in (15) is realizable and inserting $R$ to the nominal control system does not destroy the system stability.

2) Since $\left|S_{R}(j \omega)\right| \leq 1, \forall \omega \in\left[0, \omega_{b}\right]$, it follows that

$$
\begin{aligned}
|S(j \omega)| & =\left|S_{R}(j \omega) S_{N}(j \omega)\right| \\
& \leq\left|S_{N}(j \omega)\right|, \quad \forall \omega \in\left[0, \omega_{b}\right] .
\end{aligned}
$$

This implies that inserting $R$ does not compromise with amplifying the sensitivity gains at the neighboring disturbance frequencies. It should be noted that our design strategy is still subject to the waterbed effect [20], which states that the push-down sensitivity gains at the disturbance frequency are generally accompanied with the pop-up sensitivity gains somewhere else. However, in our case the amplification region is shaped to be evenly distributed along a wide high-frequency range where $Q(j \omega) \neq 1$. As such, the amplification ratio of the gain at each frequency is very small.

3) If the disturbance frequency is placed at the high-frequency region where $Q(j \omega) \neq 1$, the sensitivity gains following the disturbance frequency may be severely amplified. In this case, either the bandwidth of $Q$ should be increased or the disturbance frequency at this region should be avoided.

From the analysis above, it is important to choose an appropriate $Q(s)$ such that the frequency bandwidth of $Q$ is extended as high as possible to achieve wide-band disturbance rejection. However, in practice, this desired performance has to be compromised with sensor noise suppression. As we can see in Fig. 4, the measured pes generally contains sensor noise, then the control input would be affected by the noise amplification through the disturbance filter involving a high bandwidth $Q$ filter. In view of this tradeoff, we adopt the following $Q$ filter for the HDD track-following servo:

$$
Q=\frac{6 \tau^{2} s^{2}+4 \tau s+1}{\tau^{4} s^{4}+4 \tau^{3} s^{3}++6 \tau^{2} s^{2}+4 \tau s+1}
$$

where $\tau$ is the time constant that determines the filter bandwidth. Here, the numerator and denominator order of $Q$ are selected such that $Q$ has a best fit to unity in both gain and phase characteristics within the bandwidth. For more details of general $Q$ filter design, the interested readers are referred to [21], [22], and the references therein.

\section{EXPERIMENTAL RESULTS}

In this section, the proposed method is applied to the HDD track-following control system for disturbance rejection. We first present the nominal control system design and analyze its experimental performance, based on which the disturbance filter is thereby designed and implemented to demonstrate the improved disturbance rejection capability. To implement the controller, $S_{R}^{d}$ is discretized using a matched pole-zero method, and other control elements are discretized using a bilinear transformation [23]. Then the overall control system is implemented by 


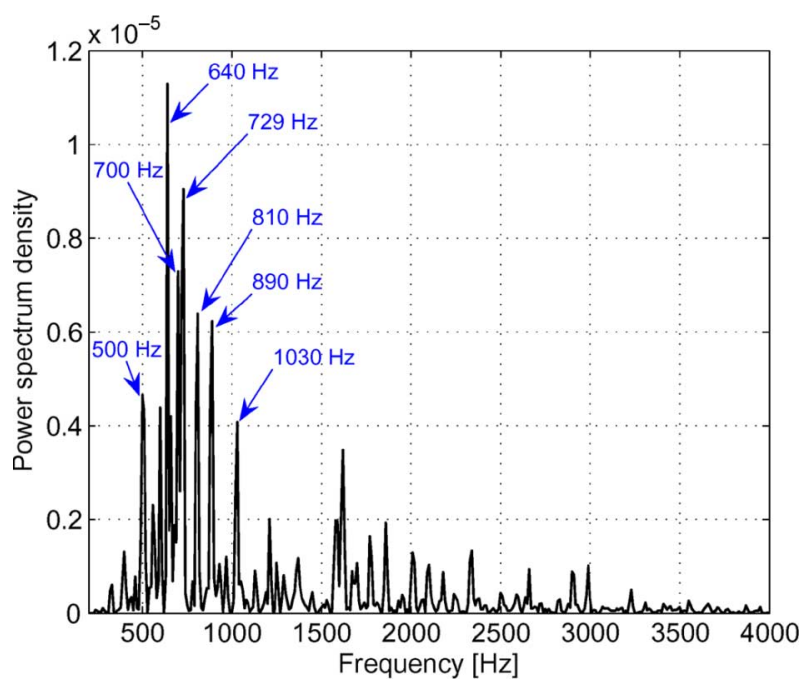

Fig. 5. Power spectra of PES under the nominal control system without disturbance filter. The arrows indicate the dominant NRROs to be rejected.

a real-time DSP system (dSPACE-DS1103). In the experiments, the head position output is measured by an LDV. The disturbance signals are collected from a real HDD with the sampling rate of $25 \mathrm{kHz}$, which consist of RROs and NRROs. We recur these disturbances in the DSP and add them to the measured head position signals following the configuration in Fig. 4. As such, only the output pes signals are available for feedback control. Such a configuration is to be consistent with a real HDD servo system to some extent.

\section{A. Nominal Controller}

The nominal controller aims at stabilizing the rigid-body mode of the VCM actuator. The typical specifications are to meet open-loop 0-dB crossover frequency $800 \mathrm{~Hz}$, phase margin $30^{\circ}$, and gain margin $5 \mathrm{~dB}$. For easy tuning, we parameterize $F$ and $L$ in terms of the servo characteristics [23] as follows:

$$
\begin{aligned}
F & =\frac{1}{k}\left[\begin{array}{ll}
\omega_{F}^{2} & 2 \zeta_{F} \omega_{F}
\end{array}\right] \\
L & =\left[\begin{array}{ll}
2 \zeta_{L} \omega_{L} & \omega_{L}^{2}
\end{array}\right]
\end{aligned}
$$

where $\zeta_{F}, \zeta_{L}$ and $\omega_{F}, \omega_{L}$ represent the damping ratio and undamped natural frequency of the system matrices $(A-B F)$ and $(A-L C)$, respectively. To achieve the desired servo specifications, we set $\zeta_{F}=\zeta_{L}=0.9, \omega_{F}=2 \pi 800$, and $\omega_{F}=2 \pi 2000$. The frequency responses of the nominal open-loop characteristics can be seen by the dashed lines in Fig. 6 while the nominal sensitivity function is also shown in Fig. 7.

\section{B. Disturbance Filter}

The nominal track-following control system is implemented. The PES samples are collected and the corresponding power spectra is shown in Fig. 5, which clearly indicates that the dominant disturbances uncompensated are in the range from 500 to $2000 \mathrm{~Hz}$, especially, at the peak frequency of 500, 640, 700, $729,810,890$, and $1030 \mathrm{~Hz}$. Therefore, the disturbance filter is designed to suppress these specific disturbances. First, we determine $S_{R}^{d}(s)$ by setting $n=7$ and $\omega_{i}$ corresponding to each peak frequency. The smaller value of $\zeta_{1 i}$ and a bigger relative
TABLE II

PARAMETERS OF $S_{R}^{d}(s)$

\begin{tabular}{|c|c|c|c|}
\hline$i$ & $\omega_{i}$ & $\zeta_{1 i}$ & $\zeta_{2 i}$ \\
\hline 1 & $2 \pi \cdot 500$ & $5 \times 10-4$ & $5 \times 10-3$ \\
\hline 2 & $2 \pi \cdot 640$ & $5 \times 10-4$ & 0.01 \\
\hline 3 & $2 \pi \cdot 700$ & $1 \times 10-3$ & $8 \times 10-3$ \\
\hline 4 & $2 \pi \cdot 729$ & $1 \times 10-3$ & $8 \times 10-3$ \\
\hline 5 & $2 \pi \cdot 810$ & $5 \times 10-4$ & $5 \times 10-3$ \\
\hline 6 & $2 \pi \cdot 890$ & $5 \times 10-4$ & $5 \times 10-3$ \\
\hline 7 & $2 \pi \cdot 1030$ & $5 \times 10-4$ & $5 \times 10-3$ \\
\hline
\end{tabular}
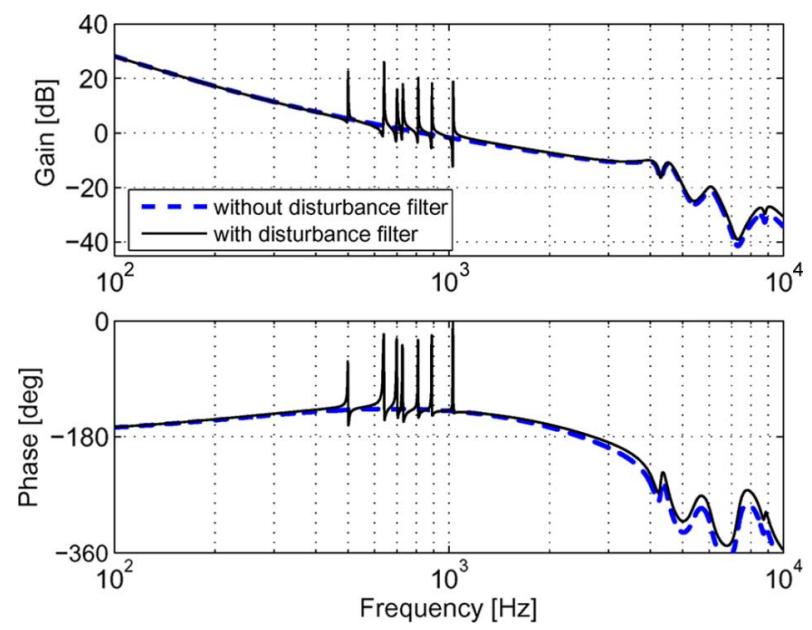

Fig. 6. Open-loop frequency responses. (Dashed lines: the nominal control system without disturbance filter; solid lines: with disturbance filter.)

ratio to $\zeta_{2 i}$ can lead to a higher reduction ratio at the disturbance frequency. In our case, the values of these parameters are listed in Table II. Second, based on the chosen $F$ and $L$ we calculate $N$ and $X$ given by

$$
\begin{aligned}
& X(s)=\frac{s^{2}+3.167 \times 10^{4} s+3.878 \times 10^{8}}{s^{2}+9048 s+2.527 \times 10^{7}} \\
& N(s)=\frac{6.4 \times 10^{7}}{s^{2}+9048 s+2.527 \times 10^{7}} .
\end{aligned}
$$

Finally, we set $\tau=1 /(2 \pi 4500)$ for the $Q$ filter. Accordingly, we can easily construct the disturbance filter $R$ from (15).

The frequency response of the open-loop system with designed disturbance filter is shown in Fig. 6, which indicates that the disturbance filter provides high gains around the target disturbance frequencies. It should be noted that although these high gains lead to multiple $0-\mathrm{dB}$ crossover frequencies, their corresponding phases are all above $-180^{\circ}$ implying that the closed-loop system is still stable. This again verifies the benefit of the factorization approach to sensitivity loop shaping with guaranteed stability. The sensitivity curve with the disturbance filter is also shown in Fig. 7 in comparison with the nominal control system without disturbance filter. It is clear that the gains at the disturbance frequencies are greatly decreased without amplifying the gains at the neighboring disturbance frequencies. 


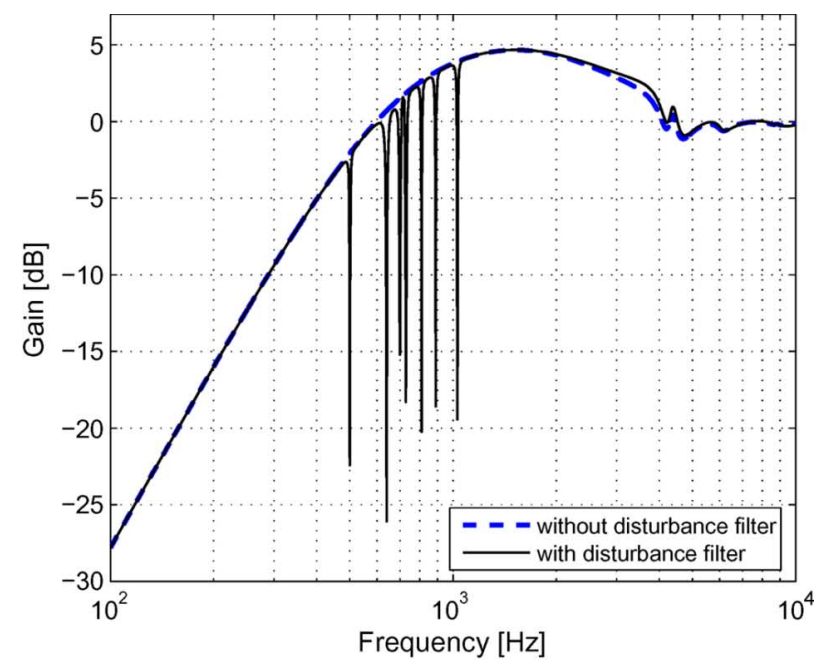

(a)

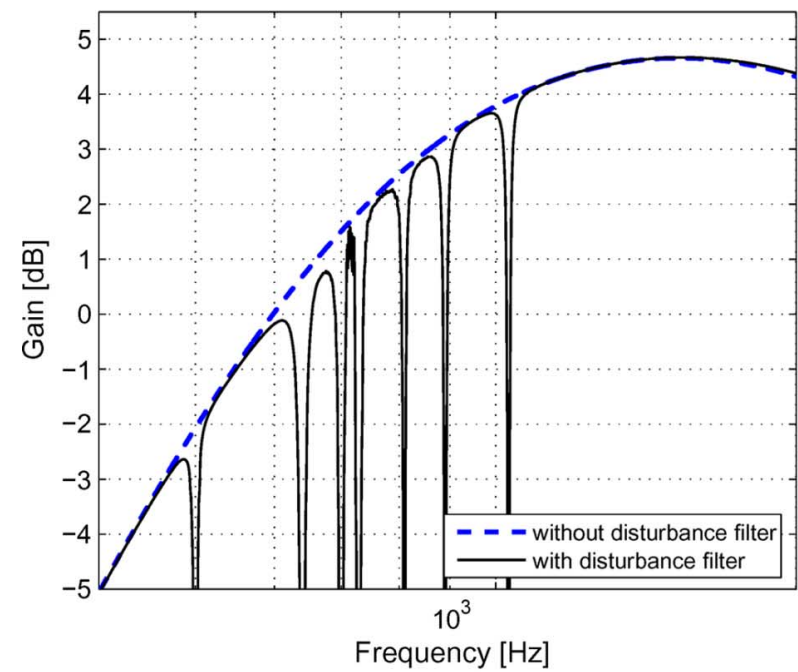

(b)

Fig. 7. (a) Frequency responses of sensitivity functions. (Dashed lines: the nominal sensitivity function without disturbance filter; solid lines: with disturbance filter.) (b) A zoomed view of (a).

Meanwhile, the slightly amplified gains only occur at the frequencies beyond $2 \mathrm{kHz}$.

The disturbance filter is inserted into the nominal control system and is implemented. Fig. 8 shows the time responses of the PES. Fig. 9 shows the power spectra of the PES with disturbance filter, which indicates that the dominant NRROs as marked in Fig. 5 have been significantly suppressed. The cumulative PES- $\sigma$ value with respect to frequency is shown in Fig. 10. These results indicate that the PES $3 \sigma$ value is reduced from $205 \mathrm{~nm}$ (without disturbance filter) to $170 \mathrm{~nm}$ (with disturbance filter), which is a $17 \%$ reduction ratio.

\section{Comparative Study}

Finally, we carry out a comparative study between the proposed disturbance filter and two other existing filters based on the classical loop shaping design, i.e., a phase-lead peak filter (PLPF) [6], [7] and a resonant filter (RF) [8]. For the sake of fair comparison, we design both comparative filters based on the same nominal controller as given in Section V-A, whose transfer
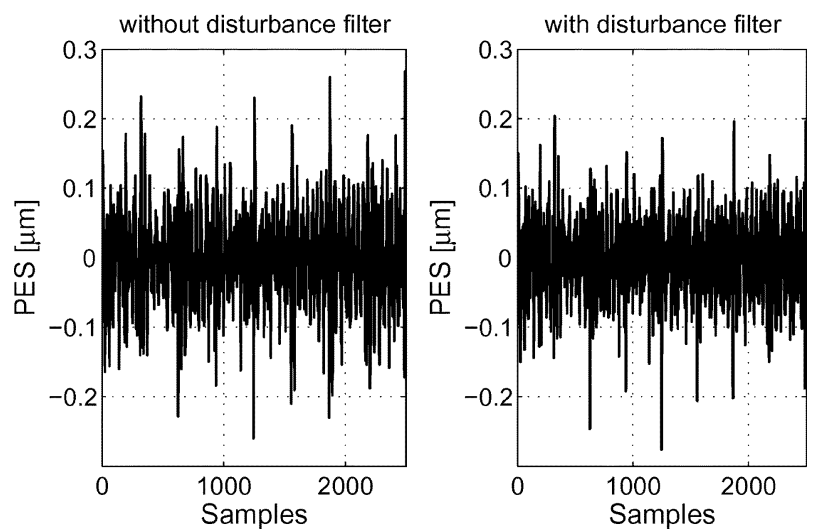

Fig. 8. Experimental results of PES time responses.

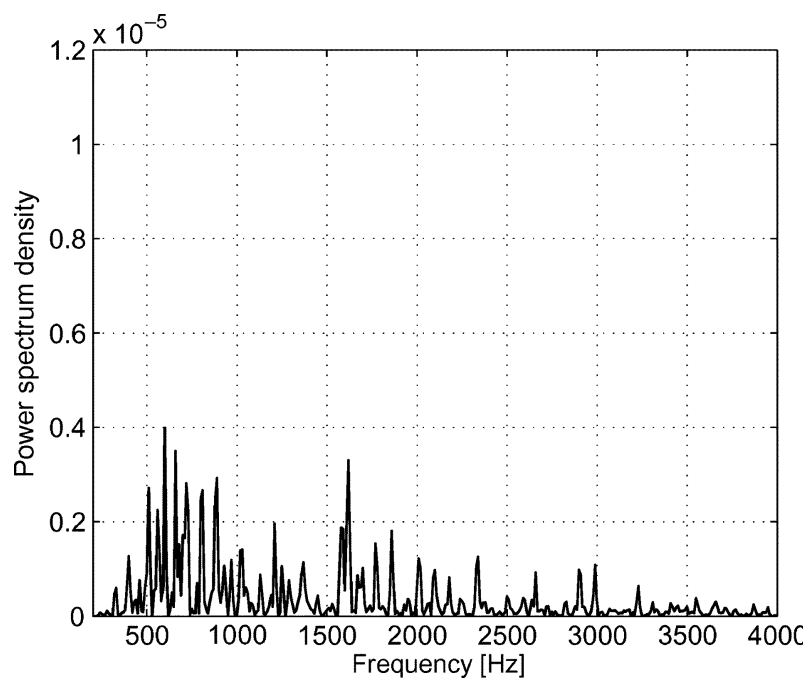

Fig. 9. Power spectra of PES with disturbance filter.

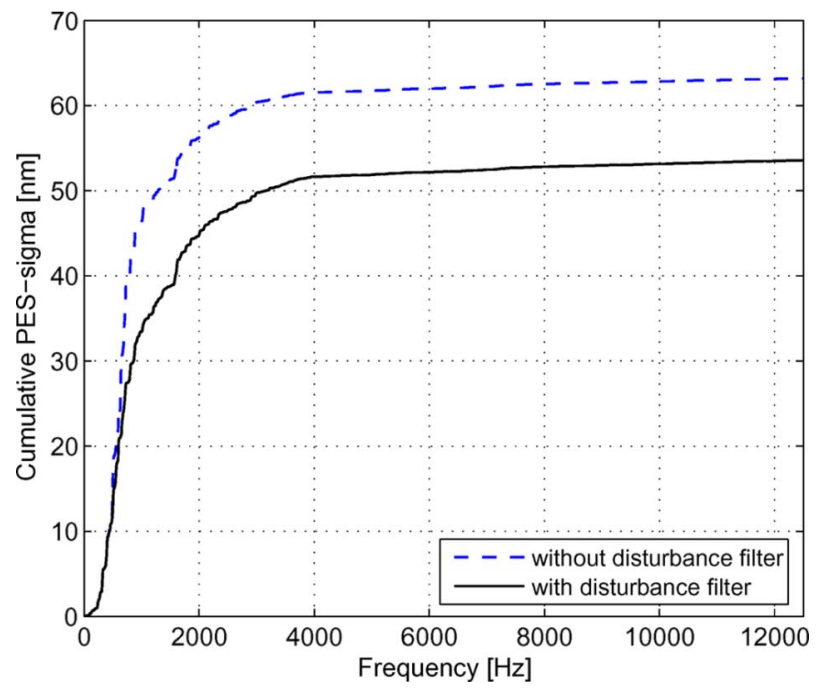

Fig. 10. Plot of cumulative PES- $\sigma$ value versus frequency.

function version can be easily calculated from (13). Additionally, both filters have been designed such that similar sensitivity characteristics are achieved at the disturbance frequencies as can be seen from Fig. 11(a). A zoomed view of the sensitivity curve 


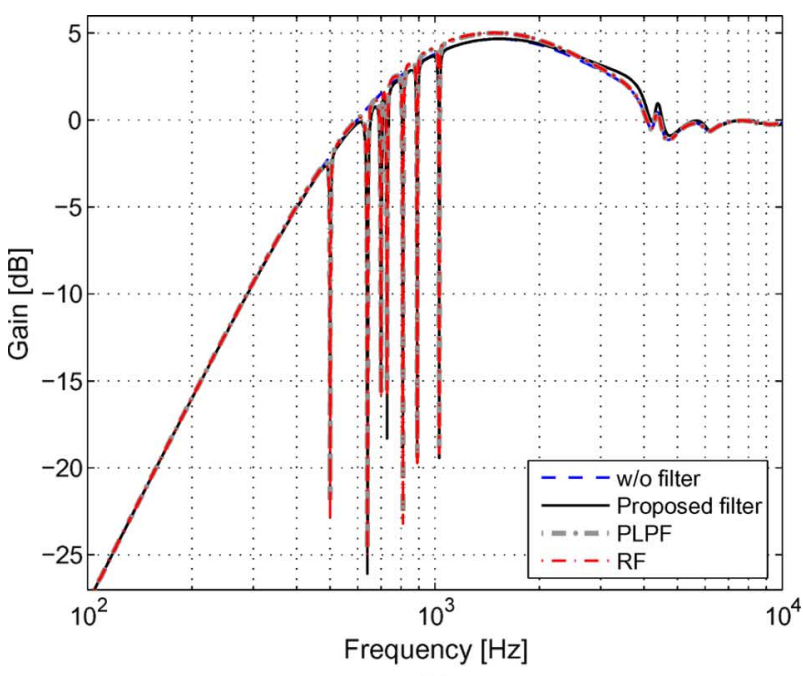

(a)

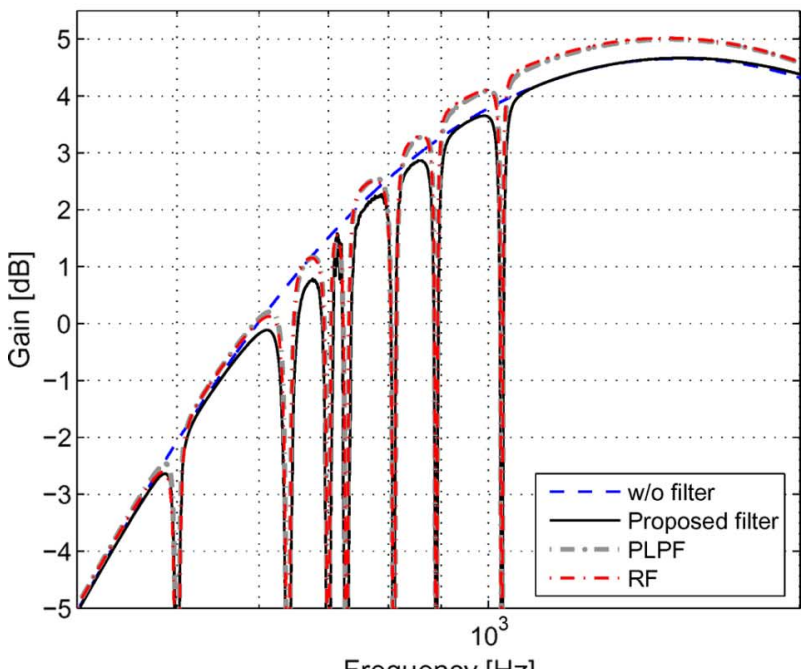

Frequency $[\mathrm{Hz}]$

(b)

Fig. 11. (a) Comparison of sensitivity functions. (b) A zoomed view of (a).

in Fig. 11(b) clearly shows that either the PLPF or the RF amplifies the sensitivity function's gains between the disturbance frequencies. The comparative filters are also implemented. Fig. 12 shows the comparisons of the PES signals. We can see that compared with the proposed filter both the PLPF and the RF result in higher peaks at the disturbance frequencies and their neighboring frequencies. As a result, both filters achieve a relatively high PES $3 \sigma$ value, PLPF: $179.5 \mathrm{~nm}$ and RF: $178.9 \mathrm{~nm}$, in comparison with the proposed filter: $170 \mathrm{~nm}$.

\section{CONCLUSION}

In this paper, we have developed a quick and effective design method for sensitivity loop shaping in HDDs. The advantage of the proposed method is that we can arbitrarily shape the sensitivity curve in terms of a disturbance filter with guaranteed stability. In addition, the disturbance filter can suppress the disturbances at some specific frequencies without amplifying the neighboring disturbances. Experimental results have demonstrated that the proposed method can remarkably suppress the disturbances around the servo bandwidth and thus re-

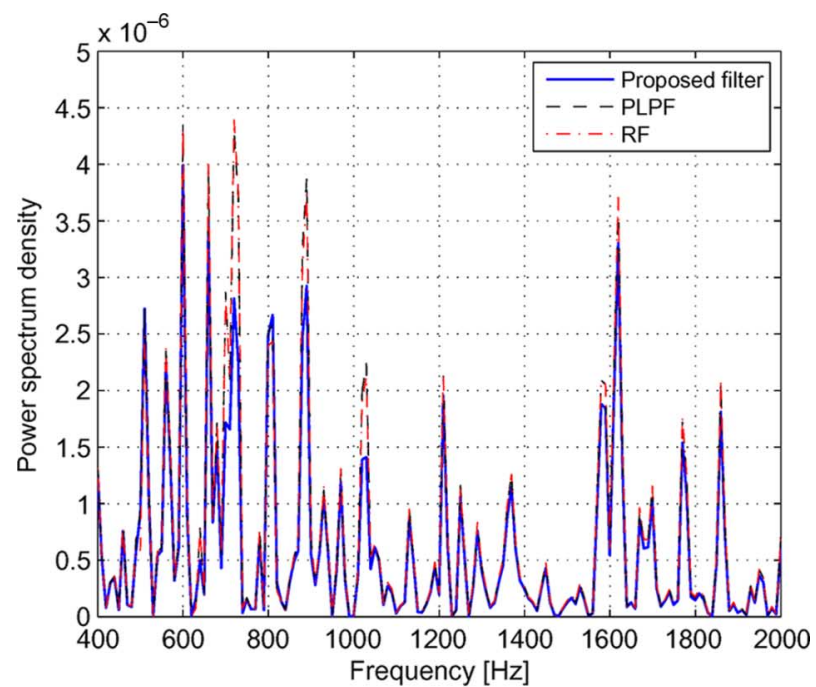

(a)

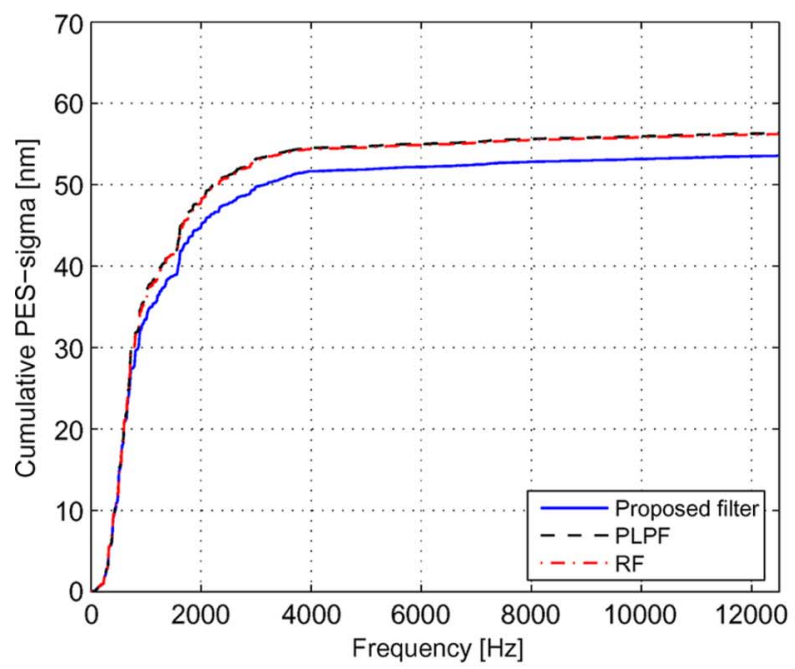

(b)

Fig. 12. Comparison of PES. (a) Power spectra. (b) Cumulative PES- $\sigma$ value.

duce the PES $3 \sigma$ by $17 \%$ based on the nominal control system. Further, a comparative study has been performed to show the proposed filter's superiority over the existing PLPF and RF.

\section{ACKNOWLEDGMENT}

This work was supported by the Australian Research Council (ARC) Center of Excellence for Complex Dynamic Systems and Control.

\section{REFERENCES}

[1] R. Wood and H. Takano, "Prospects for magnetic recording over the next 10 years," in Proc. IEEE Int. Magnetics Conf., 2006, p. 98.

[2] T. Yamaguchi, "Modelling and control of a disk file head-positioning system," Proc. Inst. Mech. Eng. I, J. Syst. Contr. Eng., vol. 215, pp. 549-567, Dec. 2001.

[3] S. M. Sri-Jayantha, H. Dang, A. Sharma, I. Yoneda, N. Kitazaki, and S. Yamamoto, "TrueTrack servo technology for high TPI disk drives," IEEE Trans. Magn., vol. 37, no. 2, pp. 871-876, Mar. 2001.

[4] M. Kobayashi, S. Nakagawa, and S. Nakamura, "A phase-stabilized servo controller for dual-stage actuators in hard disk drives," IEEE Trans. Magn., vol. 39, no. 2, pp. 844-850, Mar. 2003.

[5] J. Zheng, G. Guo, and Y. Wang, "Identification and decentralized control of a dual-actuator hard disk drive system," IEEE Trans. Magn., vol. 41, no. 9, pp. 2515-2521, Sep. 2005. 
[6] J. Zheng, C. Du, G. Guo, Y. Wang, J. Zhang, Q. Li, and B. Hredzak, "Phase lead peak filter method to high TPI servo track writer with microactuators," in Proc. American Control Conf., 2006, pp. 1309-1314.

[7] J. Zheng, G. Guo, Y. Wang, and W. E. Wong, "Optimal narrow-band disturbance filter for PZT-actuated head positioning control on a spinstand," IEEE Trans. Magn., vol. 42, no. 11, pp. 3745-3751, Nov. 2006.

[8] T. Atsumi, A. Okuyama, and M. Kobayashi, "Track-following control using resonance filter in hard disk drives," IEEE/ASME Trans. Mechatron., vol. 12, no. 4, pp. 472-479, Aug. 2007.

[9] W. Messner, "Classical control revisited: Variations on a theme," in Proc. IEEE Int. Workshop Advanced Motion Control, 2008, pp. 15-20.

[10] C. Thum, C. Du, B. Chen, E. Ong, and K. Tan, "Midfrequency runout compensation in hard disk drives via a time-varying group filtering scheme," IEEE Trans. Magn., vol. 44, no. 12, pp. 4769-4779, Dec. 2008.

[11] Y. Kim, C. Kang, and M. Tomizuka, "Adaptive and optimal rejection of non-repeatable disturbance in hard disk drives," in Proc. IEEE/ASME Int. Conf. Advanced Intelligent Mechatronics, 2005, pp. 1-6.

[12] M. Nagashima, K. Usui, and M. Kobayashi, "Rejection of unknown period disturbances in magnetic hard disk drives," IEEE Trans. Magn., vol. 43, no. 9, pp. 3774-3778, Sep. 2007.

[13] L. Guo, "Reducing the manufacturing costs associated with hard disk drives-A new disturbance rejection control scheme," IEEE/ASME Trans. Mechatron., vol. 2, no. 2, pp. 77-85, Jun. 1997.

[14] R. Ehrlich, J. Adler, and H. Hindi, "Rejecting oscillatory, non-synchronous mechanical disturbances in hard disk drives," IEEE Trans. Magn., vol. 37, no. 2, pp. 646-650, Mar. 2001.
[15] W. Messner, "Some advances in loop shaping controller design with applications to disk drives," IEEE Trans. Magn., vol. 37, no. 2, pp. 651-656, Mar. 2001.

[16] C. Du, L. Xie, J. Zhang, and G. Guo, "Disturbance rejection for a data storage system via sensitivity loop shaping and adaptive nonlinear compensation," IEEE/ASME Trans. Mechatron., vol. 13, no. 5, pp. 493-501, Oct. 2008.

[17] C. Nett, C. Jacobson, and M. Balas, "A connection between state-space and doubly coprime fractional representations," IEEE Trans. Automat. Contr., vol. AC-29, no. 9, pp. 831-832, Sep. 1984.

[18] M. Vidyasagar, Control System Synthesis: A Factorization Approach. Cambridge, MA: MIT Press, 1995.

[19] G. Guo and J. Zhang, "Feedforward control for reducing disk-flutterinduced track misregistration," IEEE Trans. Magn., vol. 39, no. 4, pp. 2103-2108, Jul. 2003.

[20] J. Doyle, B. Francis, and A. Tannenhaum, Feedback Control Theory. New York: Macmillan, 1990.

[21] A. Tesfaye, H. Lee, and M. Tomizuka, "Sensitivity optimization approach to design of a disturbance observer in digital motion control systems," IEEE/ASME Trans. Mechatron., vol. 5, no. 1, pp. 32-38, Mar. 2000.

[22] Y. Choi, K. Yang, W. Chung, H. Kim, and I. Suh, "On the robustness and performance of disturbance observers for second-order systems," IEEE Trans. Automat. Contr., vol. 48, no. 2, pp. 315-320, Feb. 2003.

[23] G. F. Franklin, J. D. Powell, and M. Workman, Digital Control of Dynamic Systems, 3rd ed. Reading, MA: Addison-Wesley, 1997. 